MASS LOSS PHENOMENA: HOT STARS -

RECENT OBSERVATIONS AND THEORETICAL IMPLICATIONS

L.B. Lucy

Department of Astronomy, Columbia University

New York, NY 10027, USA

\title{
INTRODUCTION
}

In the last decade or so, significant progress has been made in developing the theory of the winds of early-type stars. This theory, as is by now well known, is built on the basic assumption that selective radiation pressure, acting in general through a multitude of lines, is responsible both for the expulsion of matter from these stars' atmospheres and for its subsequent acceleration to beyond escape velocity (Lucy and Solomon 1970; Castor, Abbott and Klein 1975; Weber 1981). In order to investigate the effects presumed to be dominant as well as to keep within reasonable bounds the effort required to compute complete models and work out their observational implications, contributors to this line of research have concentrated on a simplified version of the general problem. Thus, for the gas dynamic part of the problem, spherically symmetric flow has usually been assumed and time-dependent effects neglected while, for the radiative transfer part, the Sobolev approximation has usually been adopted and multiple scattering effects neglected. The validity of these assumptions and approximations, and indeed of the whole enterprise, is of course ultimately a matter of observational test.

The theoretical developments cited above began when the only non-optical data pertaining to the circumstellar environments of hot stars were the first, crude far ultraviolet spectra, which were obtained with rocket-borne instrumentation (Morton 1967a, b). But now this data base has grown enormously, with highly significant contributions having come from the Copernicus, Einstein, and IUE space observatories as well as from the VLA. Much valuable data has also been acquired in the infrared, and even in the optical significant advances have been made, especially with regard to the Wolf-Rayet stars. Given this wealth of new information, it should be no surprise if our earlier ideas must now be modified. That they indeed must be is an opinion probably shared by all workers in this field. But less agreement exists on what these modifications should be. 
In addition to posing problems for the theory of line-driven, gas dynamic winds, much of this new data has been used to obtain rather persuasive empirical estimates of mass loss rates. These are obviously of great importance for investigations into the role that mass loss plays in the evolution of massive stars. But from the standpoint of this report, they are of interest only insofar as the estimated rates seem difficult to account for theoretically.

Given the existence of observational difficulties, it is of course a matter of individual judgement as to where the fault lies. Here the viewpoint will be that the theory is fundamentally sound i.e., that the basic mechanism has been correctly identified - but that certain simplifying assumptions have now been exposed as inappropriate. Specifically, great emphasis will be given to the need to drop the assumption of steady flow. Less emphasis will be given to the need to incorporate multiple scattering effects, though this technical development will surely be important in future calculations of realistic models.

Because its failures will subsequently be stressed, it is perhaps useful to recall briefly the successes of the theory of line-driven winds as developed in the 1970's. Most impressive, perhaps, is the good agreement between the domain in parameter space occupied by self-initiated wind models and that occupied by stars with detectable circumstellar absorption in far-UV resonance lines (Abbott 1979). Also persuasive is the rough agreement (for $O B$ stars) between empirical estimates of the momentum fluxes in terminal flows and the momentum fluxes transferable from photospheric radiation fields by the available line transitions (Castor, Abbott, and Klein 1976; Lamers and Morton 1976). Finally, there is the reasonable agreement between observed and predicted terminal velocities (Abbott 1978). These successes seem sufficient grounds for not readily withdrawing from selective radiation pressure the fundamental rôle it has hitherto played in theoretical developments.

As a footnote to this list of successes, it is perhaps worth emphasizing that Abbott's (1979) confirmation of an earlier demonstration (Lucy and Solomon 1970) that there exists a domain within which line-driven winds are self-initiated should dispose of an oft-repeated assertion that some other mechanism is always needed to start these outflows. This alleged general need for an initial impetus seems likely to have provided much of the appeal of models with coronal zones at the base of line-driven winds (Hearn 1975; Cassine11i, 0lson and Stalio 1978).

\section{RECENT OBSERVATIONS}

In this section, recent observational work on the circumstellar envelopes of hot stars is reviewed, with emphasis on the discoveries 
that pose problems for the theory of line-driven winds. The initial theoretical interpretations are also briefly discussed.

Superionization. Starting with Morton's (1967b) discovery of the NV resonance line in the circumstellar spectra of three Orion supergiants, spectroscopy in the far-UV has shown it to be generally true that the winds of hot stars contain stages of ionization higher than those expected if the circumstellar gas were close to being in thermal and statistical equilibrium with the photospheric radiation field, as the theory of steady, line-driven winds would predict it to be. Other ions also exhibiting this phenomenon, often referred to as superionization, are OVI and SiIV. The Copernicus data pertinent to the presence or absence of these three ions in the circumstellar envelopes of a large number of $O B$ stars has been collected and discussed by Lamers and Snow (1978).

The difficulty posed by Morton's discovery was appreciated by Lucy and Solomon (1970), and it led them to end their paper with the conjecture that the instability of a line-driven wind would generate shocks and that the resulting thermalization of a tiny fraction of the wind's bulk kinetic energy would raise the ambient temperature to $\sim 2 \times 10^{5} \mathrm{~K}$, thus allowing $\mathrm{NV}$ ions to be created by collisional ionization. A related, but far more detailed empirical model with elevated temperature was offered by Lamers and Morton (1976) as the outcome of their analysis of the Copernicus spectrum of $\zeta$ Puppis, and this became known as the warm-wind model.

An alternative way of explaining the phenomenon of superionization was proposed and worked out in considerable detail by the Wisconsin group (Cassinel1i, 0lson, and Stalio 1978; 01son 1978; Cassinelli and Olson 1979). Stimulated by the earlier work of Hearn (1975), Cassinelli and his co-workers postulated that thin zones of coronal gas exist between the photospheres of OB stars and their relatively cool, highly supersonic winds, and they went on to show that, with suitably chosen parameters, the anomalous high ions in the cool winds could then be produced by the Auger mechanism following $\mathrm{K}$-shell absorptions of X-rays emitted by the coronal gas. The Wisconsin group recognized that this model predicted X-ray fluxes from $O B$ stars that would be detectable by the Einstein Observatory, then about to be launched; they therefore computed detailed X-ray spectra to facilitate the testing of their ideas. Further refinements of this model, which came to be known as the hot corona - cool wind model, were made by 01 son and Castor (1981), who then used it to interpret the far-UV spectra of several hot stars.

$X$-ray emission. Among the earliest of the many discoveries made with the Einstein Observatory was indeed that $O B$ stars are $X$-ray emitters (Harnden et al. 1979). Moreover, the derived X-ray luminosities were close to the values predicted by the hot corona - cool wind model. Because of this quantitative success and because detectable $X$-ray emission was not a prediction that could be claimed either for the 
standard cool-wind model or for the warm-wind model, this discovery must have seemed to many investigators as decisive proof that the postulated coronal zones do indeed exist. Further examination of the $X$-ray data showed, however, that the story was not so simple.

Evidence that the $X$-ray data were not in fact consistent with the hot corona - cool-wind model, at least as originally envisaged, came when advantage was taken of the modest energy resolution provided by the IPC counts. These showed that soft X-rays ( $E<1 \mathrm{keV}$ ) were being detected, in contradiction with the severe attenuation that the Wisconsin group predicted such $X$-rays should have suffered in traversing the cool wind. This contradiction was first brought to this author's notice by Rosner and Vaiana (1979) and is discussed in the analyses of IPC data published subsequently by Long and White (1980) and by Cassinelli et al. (1981).

Although the IPC data contradict the spectra computed by Cassinelli and 01son (1979), they do not perhaps rule out somewhat modified versions of the hot corona - cool wind model. Waldron (1982), for example, claims that a realistic treatment of the transition from the hot corona to the cool wind diminishes the attenuation of soft $\mathrm{X}$-rays to an extent that makes the emergent spectrum consistent with the IPC data. Stewart and Fabian (1981), on the other hand, demonstrate that a modest reduction in mass loss rates also allows this observational contradiction to be avoided.

These modified versions of the hot corona - cool wind model are, however, contradicted by $\mathrm{X}$-ray spectra obtained with the Einstein Observatory's Solid State Spectrometer (Cassinelli and Swank 1982). The continued absence of $\mathrm{K}$-shell absorption edges at the resolution attained by this instrument would seem to eliminate all models in which $\mathrm{X}$-ray emission is confined near the base of the wind.

Absorption troughs. The existence of a problem with the absorption troughs of the P Cygni profiles of the strongest far-UV resonance lines has been evident for some time, as may be seen from the poor fits to the CIV and NV lines achieved by Lamers and Morton (1976) in their analysis of the circumstellar spectrum of $\zeta$ Puppis. The problem seems, however, to have been first expounded clearly and forcefully by Castor and Lamers (1979), from whose paper the following quote is taken: "The absorption trough in the profiles of the strongest lines, e.g., the NV 1 ine in $\zeta$ Pup, is often observed to be very nearly black over a substantial interval of wavelength, whereas the computed profiles always have a non zero intensity except just at the violet edge ..... No combination of optical depth and velocity variation can reduce this residual emission to the observed level."

Castor and Lamers (1979) briefly mention the possibility that this phenomenon could be explained with a wind that is not spherically symmetric, and this explanation can certainly be made to work in any individual case. However, since it requires a special orientation with 
respect to the observer, it cannot be accepted in view of the common occurrence of such flat-bottomed profiles. Note also that spectra of $\zeta$ Puppis taken at widely separated dates all show flat-bottomed absorption troughs.

Narrow absorption components. The above problem related to the profiles of saturated resonance lines. Yet a further problem arises when non-saturated lines are examined. These commonly show narrow, blue-shifted absorption components, again not an aspect of circumstellar spectra predictable with the theory of steady, line-driven winds.

A rather thorough discussion of these narrow absorption components has recently been published by Lamers, Gathier and Snow (1982). From Copernicus spectra of twenty-six hot stars, they conclude that such components are almost always present when observable, that they are seen typically blue-shifted by $0.75 \mathrm{v}_{\infty}$, and they arise in gas with a higher degree of ionization than the surrounding wind.

Several possible interpretations are discussed by Lamers et a1. These are all predicated, however, on these features being more or less permanent and stationary features in the spectra of these stars. This view has recently been challenged by Henrichs et al. (1982) on the basis of twenty-eight IUE spectra of $\gamma$ Cas. These reveal the formation and migration of narrow absorption components and provide the basis for Henrichs et al. to argue persuasively that with comparable coverage similar changes would be seen for other stars. On this view, these narrow absorption components represent direct evidence of time-dependent effects in these winds and thus render perhaps more plausible the finer scale time-dependence discussed in the next main section.

Mass loss rates of Wolf-Rayet stars. For many decades it has been a common conjecture that radiation pressure is somehow responsible for the WR stars' dense winds, which give rise to their characteristic and spectacular emission-line spectra. The recent efforts in developing the theory of radiatively-driven winds have, however, not yet led to dynamical models for these winds.

The difficulties facing the theoretician have become clearer in recent years as new observational data has improved the precision of estimates of both the mass loss rates and the luminosities of these stars. As the recent compilation of Barlow (1982) shows, the theoretician must contrive to produce models in which the ratio of the momentum flux in the terminal flow to that in the radiation field is in the range 10-50. A number of authors have speculated that multiple scattering effects due to the radiative coupling of neighbouring 1 ines will allow the single-scattering limit to a star's mass loss rate to be exceeded. But as yet no model has been produced demonstrating that this limit can be exceeded by a large factor. 


\section{THEORETICAL IMPLICATIONS}

In this section, it will be argued that the observational difficulties currently facing the theory of 1 ine-driven winds are largely a consequence of the assumption of steady flow. In making this point, the work done recently at Columbia on modelling time-dependent winds and computing observational implications will be briefly summarized. This work was stimulated by the previously discussed discovery of $X$-ray emission and took as its starting point the conjecture of Lucy and Solomon (1970) cited earlier.

Phenomenological models of the time-dependent winds of hot stars have been computed by Lucy and White (1980) and by Lucy (1982b). These models are attempts to represent the state attained by these winds when theix rapidly growing instabilities (Nelson and Hearn 1978; Martens 1979; MacGregor et al. 1979; Carlberg 1980; Kahn 1981) reach their limiting amplitudes. There are reasons for believing that this finite-amplitude state involves a high spatial frequency of strong, $X$-ray emitting shocks. Briefly these are: (1) the strong differential forces that arise because of shadowing in non-monotonic flow and which are available to do work to maintain shocks against dissipative losses; and (2) the rapidity with which shock-heated gas cools and thereby recovers its ability to extract momentum from the star's UV radiation field.

These phenomenological models were developed with the aim of computing the emergent $X$-ray spectra that would result from $X$-ray emitting shocks distributed throughout the winds. The outcome cannot be described as wholly successful, however. Although theoretical spectra predicted by the most recent model (Lucy 1982b) enjoy the qualitative success of predicting negligible $\mathrm{K}$-shell absorption edges, the spectra are too soft and the $\mathrm{X}$-ray luminosities too low. As a result, it is necessary to make the not implausible conjecture that there exists a spectrum of shock strengths and that the bulk of the observed $\mathrm{X}$-rays are emitted by the rare shocks of exceptional strength.

A striking confirmation of the suggestion that these winds have a high spatial frequency of shocks comes when one considers the implications of such a model for the $P$ Cygni profiles of resonance lines (Lucy 1982a). The multiply non-monotonic character that these shocks impose on the velocity field implies the existence of multiple obstacles to the continued forward propagation of radiation emitted by the photosphere. In the limit when the spatial frequency of shocks becomes infinite, no photons are forward scattered towards the observer from the wind's approaching hemisphere, and the absorption trough becomes completely black. Application of this theory to the NV 1 ine in the Copernicus spectrum of $\zeta$ Puppis (Morton 1976) shows its absorption trough to be consistent with the predicted spatial frequency of $\mathrm{X}$-ray emitting shocks. 
When this theory of 1 ine formation in non-monotronic winds is extended to resonance doublets, it turns out that further valuable information on these chaotic motions can be gleaned from their flat-bottomed absorption troughs (Lucy 1982c). As a consequence of the radiative coupling of a doublet's components, the width of the interval of zero intensity is predicted for a particular model to be $\Delta v+W$, where $\Delta v$ is the velocity separation of the components and $W$ is the amplitude of the chaotic motions in the terminal flow. Application of this theory to Morton's (1976) spectrum of $\zeta$ Puppis yields $W \simeq 500 \mathrm{~km} \mathrm{~s}^{-1}$. Thus the $U V$ line profiles provide strong evidence for a multiply non-monotonic, highly supersonic velocity field superposed on the mean outflow from $\zeta$ Puppis and indeed for other stars with flat-bottomed absorption troughs.

Although much remains unexplained, these early results in the study of time-dependent winds suffice to show that the assumption of steady flow can no longer be regarded as appropriate. It is to be expected that some at least of the remaining difficulties will also be explained as time-dependent effects are more thoroughly investigated.

Work at Columbia on stellar winds is supported by the National Science Foundation under grant NSF AST 81-15971. The final version of this report was written during a sabbatical leave spent at the European Southern Observatory, Munich. I am grateful to Prof. L. Woltjer for the invitation to spend this time at ESO.

\section{REFERENCES}

Abbott, D.C. 1978, Ap.J., 225, 893.

Abbott, D.C. 1979, in IAU Symposium No. 83, ed. P.S. Conti and C.W.H. de Loore (Holland: Reidel), p. 237.

Barlow, M.J. 1982, in IAU Symposium No. 99, ed. C.W.H. de Loore and A.J. Will is (Holland: Reide1), p. 149.

Carlberg, R.G. 1980, Ap.J., 241, 1131.

Cassinel1i, J.P., olson, G.L., and Stalio, R. 1978, Ap.J., 220, 573. Cassinel1i, J.P., and Olson, G.L. 1979, Ap.J., 229, 304 .

Cassine11i, J.P., Waldron, W.L. Sanders, W.T., Harnden, F.R., Jr., Rosner, R., and Vaiana, G.S. 1981, Ap.J., 250, 677.

Cassinelli, J.P., and Swank, J.H. 1982, preprint.

Castor, J.I., Abbott, D.C., and Klein, R.I. 1975, Ap.J. 195, 157.

Castor, J.I., Abbott, D.C., and Klein, R.I. 1976, in Physique des mouvements dans les atmosphères stellaires, ed. R. Cayrel and M. Steinberg (Paris: CNRS), p. 363 .

Castor, J.I., and Lamers, H.J.G.L.M. 1979, Ap.J.Suppl., 39, 481. Harnden, F.R., et al. 1979, Ap.J. (Letters), 234, L51.

Henrichs, H.F., Hammerschlag-Hensberge, G., Howarth, I.D., and Barr, P. 1982, Ap.J., in press.

Kahn, F.D. 1981, M.N.R.A.S., 196, 641.

Lamers, H.J.G.L.M., and Morton, D.C. 1976, Ap.J.Suppl., 32715.

Lamers, H.J.G.L.M., and Snow, T.P. 1978, Ap.J., 219, 504. 
Lamers, H.J.G.L.M., Gathier, R., and Snow, T.P. 1982, Ap.J., $258,186$.

Long, K.S., and White, R.L. 1980, Ap.J. (Letters), 239, L65.

Lucy, L.B., and Solomon, P.M. 1970, Ap.J., 159, 879.

Lucy, L.B., and White, R.L. 1980, Ap.J., 241, 300.

Lucy, L.B. 1982a, Ap.J., 255, 278.

Lucy, L.B. $1982 \mathrm{~b}, \overline{\mathrm{Ap} . \mathrm{J}} ., 255,286$.

Lucy, L.B. 1982c, $\overline{\text { Ap.J. }}$, submitted.

MacGregor, K.B., Hartmann, L., and Raymond, J.C. 1979, Ap.J., 231,514 .

Martens, P.C.H. 1979, Astr. Ap., 75, L7.

Morton, D.C. 1967a, Ap.J., 147, 1017.

Morton, D.C. $1967 \mathrm{~b}, \overline{\mathrm{Ap} . \mathrm{J}} ., 150,535$.

Morton, D.C. 1976, AP.J. $, 203,386$.

Nelson, G.D., and Hearn, A.G. 1978, Astr. Ap., 65, 223.

Olson, G.L. 1978, Ap.J., 226, 124.

Olson, G.L., and Castor, J.I. 1981, Ap.J., 244, 179.

Rosner, R., and Vaiana, G.S. 1979, private communication.

Stewart, G.C., and Fabian, A.C. 1981, M.N.R.A.S., 197, 713.

Waldron, W.L. 1982, Wisconsin Preprint, No. 148.

Weber, S.V. 1981, Ap.J., 243, 954. 